\title{
Variation of Peak Expiratory Flow Rate with Body Mass Index in Medical Students of Karachi, Pakistan
}

\author{
Hasnain Abbas Dharamshi, \\ Ahmad Faraz ${ }^{1}$, Erum Ashraf ${ }^{2}$, Ali Abbas Mohsin Ali $^{3}$, \\ Mansoor Ali Merchant Rameez ${ }^{4}$ \\ Syed Muhammad Ali Abidi ${ }^{3}$, Saad Ullah ${ }^{4}$, \\ Mustaqueem Ur Rehman ${ }^{5}$, Taha Nafees ${ }^{1}$
}

\section{Abstract}

Objective: The primary aim of our study was to assess the variation of PEFR with BMI in normal medical students of Karachi, Pakistan.

Design: Cross-sectional study.

Setting: Medical students of Karachi Medical and Dental College.

Participants: 138 non-smoker healthy medical students composed of 111 females and 27 males.

Variable Parameters: They include mean age, body height and body weight and PEFR. They were marked separately for each gender.

Results: The mean BMI in females was found out to be $18.54 \pm 2.10$ corresponding with that of mean PEFR value $431.62 \pm 56.62$ whereas in males the mean BMI was $25.07 \pm 2.96$ corresponding with that of mean PEFR value $533.70 \pm 23.22$. Also there is a statistically significant variation in PEFR with an increase in BMI.

Conclusion: The study concludes that PEFR is affected positively by variation in BMI. Also young males have more BMI and PEFR values than their young female counterparts. A large sample size with accurate peak flow meter is required along with ethnic consideration of the study population for better and accurate results.
1 Karachi Medical and Dental College.

2 Jinnah Medical and Dental College.

3 Sindh Medical College, Dow University of Health Sciences.

4 Dow Medical College.

5 Baqai Medical College.

Contact information:

Hasnain Abbas Dharamshi.

Address: Soldier bazaar 2, 74000,

Karachi, Pakistan.

Tel: 923312729129.

झdr.hasnain.dh@hotmail.com

Keywords

PEFR, B.M.I, Medical Students. 


\section{Introduction}

The WHO classifies an individual as being "overweight" or "obese" based on their body mass index (BMI), which measures weight relative to height. According to $\mathrm{WHO}$, a person having $\mathrm{BMI}$ $\geq 25 \mathrm{~kg} / \mathrm{m}^{2}$ shall be considered as overweight while obese is defined as having a BMI $\geq 30 \mathrm{~kg} / \mathrm{m}^{2}$. However, these values are rough estimate because they may not accurately correspond to the same degree of fatness in different individuals. [1]

Obesity stands out as an emerging global public health challenge that is epidemically increasing in both developed and underdeveloped countries. The WHO ranks Pakistan as 165 (out of 194 countries) in terms of its overweight population, with $22.2 \%$ of individuals over the age of 15 crossing the threshold level of obesity. [2]

A research study conducted at Ain Shams University concluded that about 50 percent of medical students of that University were overweight and obese.3 The most significant factors they found responsible for obesity in medical students include genetic factors and life style factors like prolong computer sitting on daily basis, overeating during time of stress and eating between meals. [3] Also consumption of energy dense fast foods that are readily available in the university cafeteria play a significant role in adding up to the obesity of a typical university student. [4]

The overweight and obese people are at high risk for developing many different co-morbidities and health conditions, including respiratory disease. [5] It happens because obesity stiffens the total respiratory system presumably due to its combined effects on lung and chest wall compliance leading to respiratory problems, such as breathlessness, specifically during exercise, even if they are devoid of any respiratory illness. [6]

Peak expiratory flow rate (PEFR) signifies the maximum flow that is achieved during forceful expiration after maximum inhalation. It is an important tool to identify the degree of obstruction in the airways by measuring air flow through the bronchi. PEFR is influenced by various factors such as age, height, weight, gender, race and the environmental conditions. [7] Although it's an established fact that forced expiratory volume over $1 \mathrm{sec}\left(\mathrm{FEV}_{1}\right)$ is more efficient and accurate than PEF for indicating airway obstruction. However the PEF, due to its portability, cost effectiveness, feasibility and simplicity of maneuver, has made it preferable over FEV ${ }_{1}$. [8] The Mini Peak Flow Meter is a useful instrument that is widely used for ambulatory PEF measurement. [8]

Considering the overwhelming rising burden of obesity among the youth, this research was conducted on undergraduate medical students as they are the future of the health care system and therefore their health status is necessary for effective functioning of the health sector. This study primarily aimed to assess the respiratory system functionality in medical students through variation of PEFR values with that of BMI.

Further studies are required to categorize different values of PEFR in relation to ethnicity so that those values can be considered in the evaluation of normal PEFR values while considering variation with other influencing factors.

\section{Materials And Methods}

This cross-sectional study was carried out on 138 medical students currently studying in Karachi Medical and Dental College. All the participants were non-smoker healthy young Pakistani medical students between the ages of 18 and 25. Students that were not enrolled in the study include those that were regular exercise takers or those who had some respiratory illness and were taking treatment for the same. The variable parameters of this study include mean age, body height and body weight and PEFR. They were marked separately for each gender. Prior to the experiment, every participant was individually informed about the experimental protocol as follows; 
- Take deep breath before blowing it forcefully into the mouth pieces of the Mini Wright Peak flow meter

- Nostrils should be closed quickly and tightly while blowing into the mouth pieces of the instrument.

- Tongue shouldn't be kept in front of the mouthpiece

- Every participant should perform the activity thrice

- The experiment needs to be started again in case of coughing or sneezing while breathing out

- Tight clothing should be avoided

On the experimental day, all the subjects were made to relax physically and mentally while being seated. Then their vitals such as blood pressure, heart rate and respiratory rates were recorded and PEFRs were measured within careful observation of the researcher. The standing height and weight were measured with shoes taken off. The standing heights were measured in centimeter using standard height measuring rods and weights were measured in kilograms with calibrated Libra weighing scale. The PEFR was noted in liters/minute with the aid of Mini Wright Peak Flow Meter. The study was conducted from August 2013 to December 2013.

Every participant was involved thrice in the activity for PEFR values with all protocols and the highest value was considered as the value of PEFR for that individual. Similar pattern was followed for categorizing the subjects according to their weight. P-value of less than or equal to 0.05 ( $p \leq 0.05$ ) was considered as statistically significant. SPSS 17 was used for statistical analysis of all the data that was acquired for this study.

\section{Results}

A total of 138 undergraduate medical students were enrolled in this study. Table I demonstrate the distribution of the study population with females forming majority of the participants 80.4 $\%$ having the mean age $18.68 \pm 0.83$. Males the minority $19.6 \%$ of subjects with mean age of $18.33 \pm 0.78$.

\section{Table 1.}

\begin{tabular}{|l|c|c|}
\hline & Frequency & Percent \\
\hline Male & 27 & 19.6 \\
\hline Female & 111 & 80.4 \\
\hline Total & 138 & 100.0 \\
\hline
\end{tabular}

Table $\mathbf{2}$ and $\mathbf{3}$ gives information about the values of BMI with their corresponding values for PEFR in females and males respectively. It can be noted here that there is a statistically significant variation in PEFR with an increase in BMI.

Table 2. Variation of BMI with PEFR in females.

\begin{tabular}{|l|c|c|}
\hline \multicolumn{1}{|c|}{ BMI } & No Of Persons & PEFR (Mean \pm S.D) \\
\hline$<18.5$ & 54 & $386.58 \pm 45.67$ \\
\hline $18.5-24.9$ & 53 & $471.51 \pm 20.76$ \\
\hline $25-29.9$ & 4 & $511.25 \pm 11.08$ \\
$>30$ & 0 & $00.00 \pm 00$ \\
\hline
\end{tabular}

Correlation coefficient $(r)=.778, N=111$

Table 3. Variation of BMI with PEFR in males.

\begin{tabular}{|l|c|c|}
\hline \multicolumn{1}{|c|}{ BMI } & No Of Persons & PEFR $($ Mean \pm S.D) \\
\hline$<18.5$ & 0 & $00.0 \pm 00$ \\
\hline $18 .-24.9$ & 16 & $381.56 \pm 29.98$ \\
\hline $25-29.9$ & 7 & $500.00 \pm 73.20$ \\
$>30$ & 4 & $546.25 \pm 19.73$ \\
\hline
\end{tabular}

Correlation coefficient $(r)=.832 \mathrm{~N}=27$

The mean BMI in females was found out to be $18.54 \pm 2.10$ corresponding with that of mean PEFR value $431.62 \pm 56.62$ whereas in males the 
Table 4. Summary of the research data.

\begin{tabular}{|l|c|c|c|c|}
\multicolumn{1}{|c|}{ Subjects } & Age In Years & PEFR $($ Mean \pm S.D) & PEFR (Mean \pm S.D) & PEFR (Mean \pm S.D) \\
\hline Males (27) & $18.33 \pm 0.78$ & $25.07 \pm 2.96$ & $533.70 \pm 23.22$ & .832 \\
\hline Females (111) & $18.68 \pm 0.83$ & $18.54 \pm 2.10$ & $431.62 \pm 56.62$ & .778 \\
\hline P Value & $<0.0001^{*}$ & $<0.0001^{*}$ & $<0.0001^{*}$ & $<0.0001^{*}$ \\
\hline
\end{tabular}

mean BMI was $25.07 \pm 2.96$ corresponding with that of mean PEFR value $533.70 \pm 23.22$ as narrated by Table 4 .

\section{Discussion}

This study reported interesting relationship between BMI and PEFR values with increasing trend in the values of latter with the increase in the former values. Similar finding was reported by Sudha. D [9]. However this relationship of BMI with PEFR was contrasted by the results given by Kaur Harpreet7 and Saraswathi llango [10]. The ambiguity of increase in PEFR with rise is BMI above normal limit of 24.9 can be addressed by increasing the sample size of people with abnormal BMI values (BMI $\geq 25)$ comparable to the no of participants with normal BMI as our research study included few participants with abnormal BMI values.

Consistent with other research studies, [11, 12] the mean BMI values were found out to be greater in male participants than the female participants, although the values were comparably lower than American medical college students. [13] Also it was found that a significant majority $48.6 \%$ of the female participants had BMI lower than 18.5. The PEFR values, due to their relation with $\mathrm{BMI}$, were also found out to be higher in males than the females similar to other studies. [12, 14]

A large sample size is needed to get more accurate results. The ethnicity also needs to be considered as it is an important factor that affects the values of PEFR.[7] Moreover the Mini Wright Peak Flow Meter is less accurate than its counterparts so therefore a more accurate peak flow meter can level up the accuracy of the acquired data.[15]

\section{Conclusion}

The study concludes that PEFR is affected positively by variation in BMI. Also young males have more $\mathrm{BMI}$ and PEFR values than their young female counterparts. A large sample size with accurate peak flow meter is required along with ethnic consideration of the study population for better and accurate results.

\section{References}

1. World Health Organisation. Fact sheet: obesity and overweight. March 2011. Available at: http://www.who.int/mediacentre/ factsheets/fs311/en/print.html. Accessed 10 March 2014.

2. Streib, Lauren (2 August 2007). "World's Fattest Countries". Forbes

3. Bakr EM, Ismail NA, and Mahaba HM. Impact of life style on the nutritional status of medical students at Ain Shams University. The Journal of the Egyptian Public Health Association. 2001; 77, no. 1-2: 29-49

4. Ganasegeran K, Al-Dubai SA, Qureshi AM, Al-abed AA, Am R, Aljunid SM. Social and psychological factors affecting eating habits among university students in a Malaysian medical school. Nutr J, 2012; 11.48: 107.

5. Gundogdu Z, Eryilmaz N. Correlation between peak flow and body mass index in obese and non-obese children in Kocaeli, Turkey. Prim Care Respir J. 2011; 20(4): 403-406

6. Salome CM, King GG \& Berend N. Physiology of obesity and effects on lung function. Journal of Applied Physiology.2010; 108(1): 206-211

7. Harpreet K, Jagseer S, Manisha M, Khushdeep S, Ruchika G. Variations in the Peak Expiratory Flow Rate with Various Factors in a Population of Healthy Women of the Malwa Region of Punjab, India. Journal of Clinical and Diagnostic Research. 2013; 7(6): 1000-03. [PMC free article] [PubMed] 
8. Mittal S, Gupta S, Kumar A, Singh KD. Regression equations for peak expiratory flow in healthy children aged 7 to 14 years from Punjab, India. Lung India. 2013 Jul-Sep; 30(3): 183-186.

9. Sudha D, Chandra SE, Saikumar P. Correlation of Nutritional Status and Peak Expiratory Flow Rate in Normal South Indian Children Aged 6 to 10 Years. IOSR JDMS. Nov- Dec 2012; 2(3): 11-16

10. Saraswathi I, Christy A, Saravanan A, Dr Prema S. Correlation of Obesity Indices with Peak Expiratory Flow Rate in Males and Females. IOSR Journal of Pharmacy. February - 2014; 4(2): 21-27

11. Jie C, He Y, Yacun F, et al. Factors associated with being overweight among Inner Mongolia medical students in China. BMJ open. 2013; 3(12) bmjopen.bmj.com/content/3/12/ e003900.abstract

12. Joffa PK, Nwafor A and Adienbo OM. Correlation between Body Mass Index and Peak Expiratory Flow Rate of an Indigenous Nigerian Population in the Niger Delta Region. Res. J Recent Sci. February 2013; 2(2): 28-32

13. Sira N, Pawlak R. Prevalence of overweight and obesity, and dieting attitudes among Caucasian and African American college students in Eastern North Carolina. Nutr Res Pract. 2010; 4: $36-42$

14. Ali Baig MI, Qureshi RH. Pulmonary function tests: normal values in non-smoking students and staff at the Aga Khan University, Karachi. J Coll Physicians Surg Pak. 2007 May; 17(5): 265-8

15. Miller MR, Dickinson SA, Hitchings DJ. The accuracy of portable peak flow meters. Thorax. Nov 1992; 47(11): 904

\section{Comment on this article:}

\section{$f(B)$ in $8+S P$}
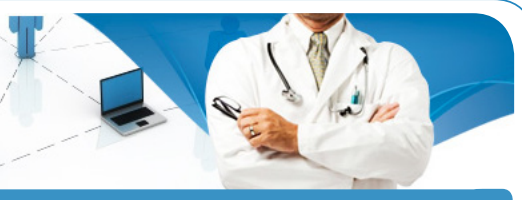

http://medicalia.org/

Where Doctors exchange clinical experiences, review their cases and share clinical knowledge. You can also access lots of medical publications for free. Join Now!

\section{Publish with iMedPub}

http://www.imed.pub

International Archives of Medicine is an open access journal publishing articles encompassing all aspects of medical science and clinical practice. IAM is considered a megajournal with independent sections on all areas of medicine. IAM is a really international journal with authors and board members from all around the world. The journal is widely indexed and classified Q1 in category Medicine. 